\title{
Landscape Design: Ten Important Things to Consider ${ }^{1}$
}

Gail Hansen²

When designing a residential landscape, the most important step is to put a plan on paper. Developing a master plan will save you time and money and is more likely to result in a successful design. A master plan is developed through the 'design process'; a step-by-step method that considers the environmental conditions, your desires, and principles of design. The goal is to organize the natural and man-made features in your yard into an aesthetic, functional, and environmentally sustainable landscape.

\section{The Design Process}

The five steps of the design process include: 1) conducting a site inventory and analysis, 2) determining your needs, 3) creating functional diagrams, 4) developing conceptual design plans, and 5) drawing a final design plan. The first two steps establish the aesthetic, functional, and horticultural requirements for the design. The last three steps then apply those requirements to the creation of the final design.

The process begins with a site inventory and analysis of soil, drainage, climate conditions, and existing vegetation. This is a critical step for both plant selection and placement, and locating family activities and functions.
It's important because the same climate conditions that affect the plants - temperature, humidity, rain, wind, and sunlight - also affect you, the user. The next step is to make a list of your needs and desires- this helps you determine how your yard and landscape will be used. The site and user analyses will also help you establish a theme for the form and style of your design. The functional diagram is then used to locate the activity spaces on the site and from this diagram a conceptual plan is developed. The last step is a final design that includes all the hardscape and planting details that are necessary for installation. Throughout the design process there are ten important things to consider:

1. Understand your site for plant selection and activity location

2. Remember the user by considering what you want and need

3. Use a form and/or style theme to help determine shapes and organize spaces

4. Create and link spaces by designating activity areas and linking with elements

5. Consider the function of plants for both the environment and the user

6. Structure the plantings by using massing and layering techniques

1. This document is ENH1112, one of a series of the Environmental Horticulture Department, Florida Cooperative Extension Service, Institute of Food and Agricultural Sciences, University of Florida. Original publication date January 2009. Visit the EDIS Web Site at http://edis.ifas.ufl.edu.

2. Gail Hansen, assistant professor, Environmental Horticulture Department, Center for Landscape Conservation and Ecology; Florida Cooperative Extension Service, Institute of Food and Agricultural Sciences, University of Florida, Gainesville, FL 32611. 
7. Highlight important points such as transition areas and focal points

8. Pay attention to detail in the materials, the colors, and the surface textures

9. Take time into account for the growth and maintenance of plants

10. Protect your resources by using sustainable design practices

\section{Understand Your Site}

A thorough inventory and analysis of the site is important to determine the environmental conditions for plant growth and the best use of the site. Issues of concern include the soil type, topography, and regional climate. The type of soil determines the nutrients and moisture available to the plants. It is always best to use plants that will thrive in the existing soil. Although soil can be amended, amendment is often costly and most times ineffective. Existing vegetation can provide clues to the soil type. Where plants grow well, note the soil conditions and use plants with similar growing requirements. Pay particular attention to areas where plants are not doing well and adjust when choosing new plants. Topography and drainage should also be noted and all drainage problems corrected in the proposed design. A good design will move water away from the house and reroute to other areas of the yard.

Climate concerns begin with temperature: plants must be able to survive the average high and most importantly, the average low temperatures for the region. Use the USDA Plant Hardiness Zone Map

(www.usna.usda.gov/Hardzone/) as a starting point to choose plants appropriate for your zone, but remember microclimates within your yard can offer alternatives. Sun/shade patterns, the amount and length of exposure to sun or shade (Figure 1) create microclimates (sometimes called microhabitats).

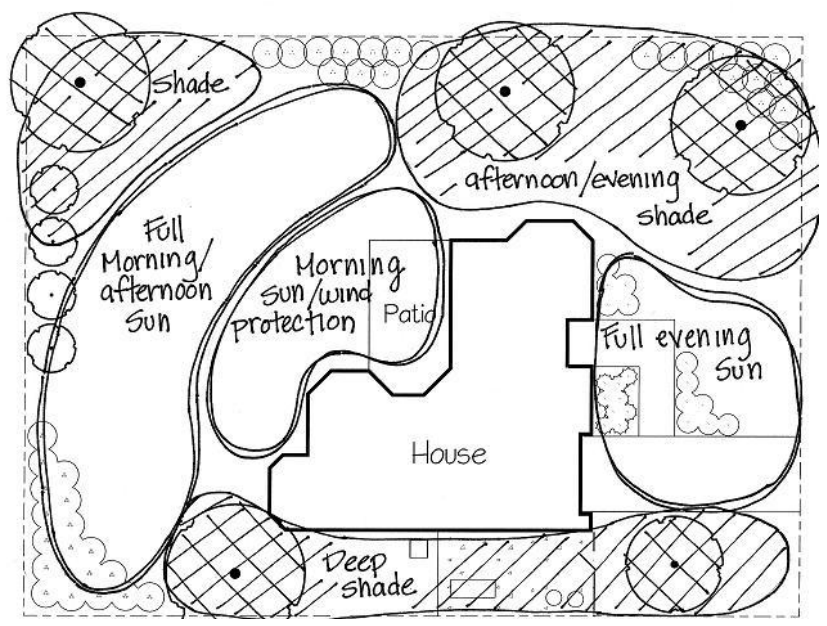

Figure 1. Sun/Shade Patterns. Credits: Gail Hansen

Recording site conditions and existing vegetation on a base map will reveal the location of microclimates in the yard. Plants usually fall into one or two of four microclimate categories- full sun, partial shade, shade and deep shade. It is important to remember that sun/shade patterns change with the seasons and over time as trees get larger.

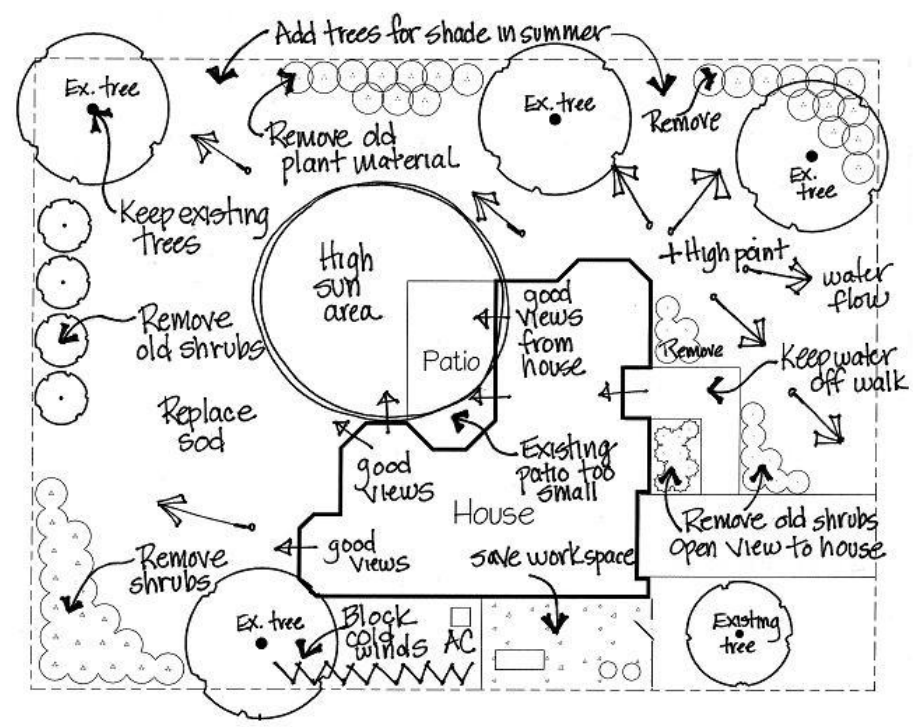

Figure 2. Site Inventory. Credits: Gail Hansen

It is essential to note all the existing conditions on an accurate base map when doing the site inventory (Figure 2). Utilities such as power lines, septic tanks, underground utilities and roof overhangs determine plant location. Use a surveyor's plat of your property for the boundaries and location of your home. Measure 
and note on the survey other structures and hardscape such as a patio, driveway, or sidewalk. It is very important to hire a surveyor if you do not have a plat; guessing the location of boundaries can be a costly mistake.

\section{Remember the User}

The users are typically you, your family, the family pets, and visitors, and each have their own needs. There are five things to consider: 1 ) how do you currently use the yard, 2) how do you want to use the yard, 3) aesthetically, how do you want it to look, 4) what is your maintenance style, hands-off or hands-on, and, 5) what is your budget.

It is very important to consider how you currently use the yard. For example- which entry is used by whom, where do the kids play and where does the dog usually run? (Figure 3)

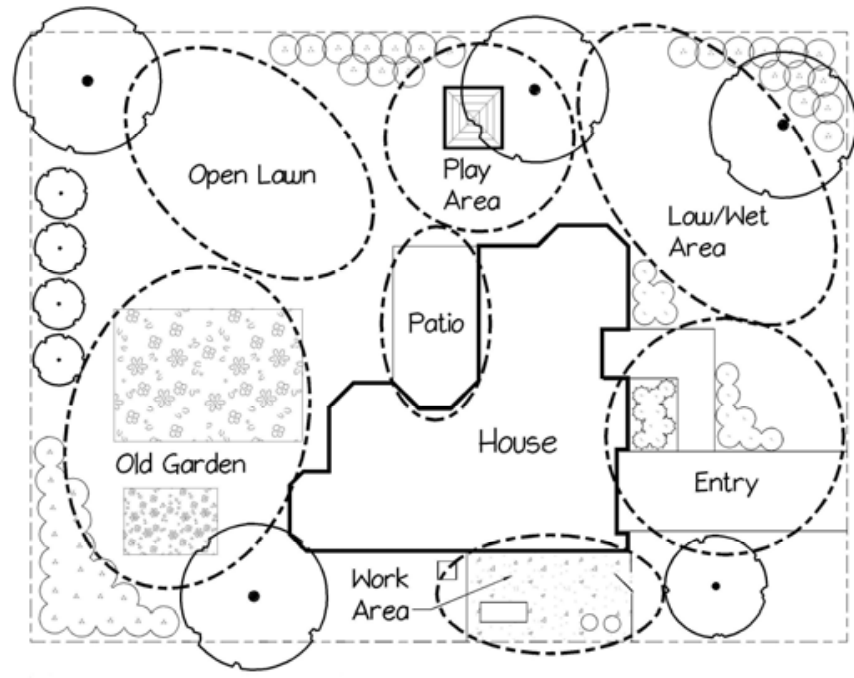

Figure 3. Current Use Areas. Credits: Gail Hansen

Thinking about how you currently use the yard, and how you want to use it in the future (Figure 4), determines the need to re-organize old spaces into new spaces and amenities.

It is also important to remember the vehicles used by your family; driveways and parking are space intensive. Budget concerns include the materials, initial installation costs and the on-

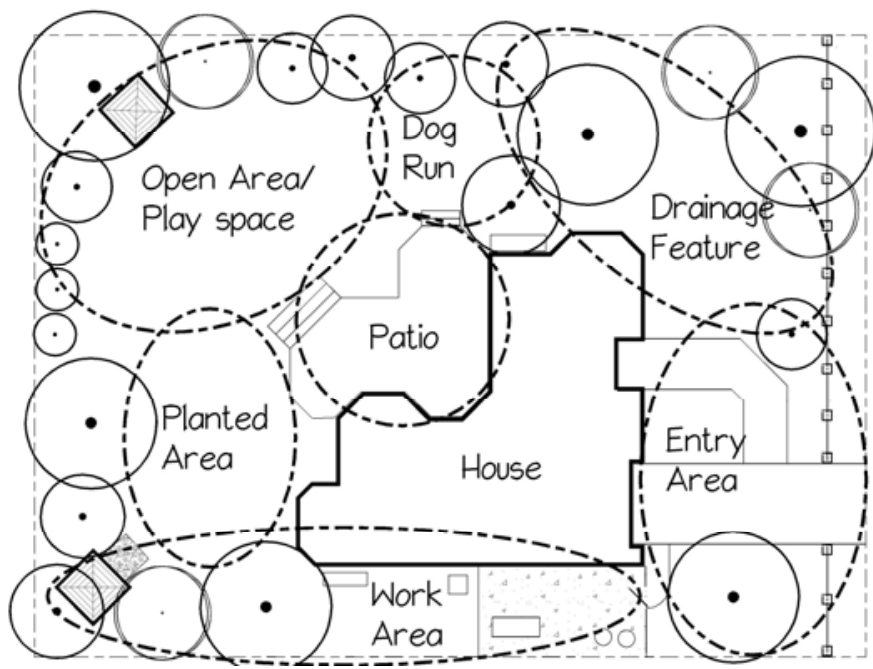

Figure 4. Proposed Use Areas. Credits: Gail Hansen

going maintenance costs. Determine the time and money you are willing to put into maintaining the plants and hardscape-be realistic about your intentions and ability.

\section{Use a Form and/or Style Theme}

There are many different landscape themesfrom simple to complex, but it is helpful to choose one to guide your plant and material selection. Think of a theme as the inspiration for your garden. Many people find it helpful to look in gardening magazines and books for ideas. This is a good start, but be aware that the gardens in the photos were picked because they are outstanding examples. Look at the photos with a critical eye to gather ideas that you can adapt to your passion level, your budget and your site. Before choosing a theme it is important to look at the surrounding views of your property. Decide if you want to open your yard, close your yard, or a little of both, to these views. In other words, do you want the garden to enclose the space around you and relate mostly to the house, or do you want the garden to open views and look outward, relating to the surroundings? This will give you a starting point to think about a theme. Care should be taken to choose appropriate themes for your yard based on the architecture, the type of neighborhood, the topography, and the regional 
landscapes. This is called "sense of place", which means it fits with the surroundings.

There are both form themes and style themes. Every garden should have a form theme, but not all gardens have a style theme. In fact, many residential gardens have no particular style except to blend with the house by repeating details from the architecture such as materials, color, and form. All gardens, however, should use a form theme to create spaces for activities. In a form theme the organization and shape of the spaces in the yard is based either on the shape of the house, the shape of the areas between the house and the property boundaries, or a favorite shape of the homeowner. The form theme determines the shape and organization (the layout) of the spaces and the links between them. Common themes include geometric, such as a circle, square, and rectangle, or naturalistic, such as irregular (organic edge) or curvilinear (meandering lines) (Figure 5).

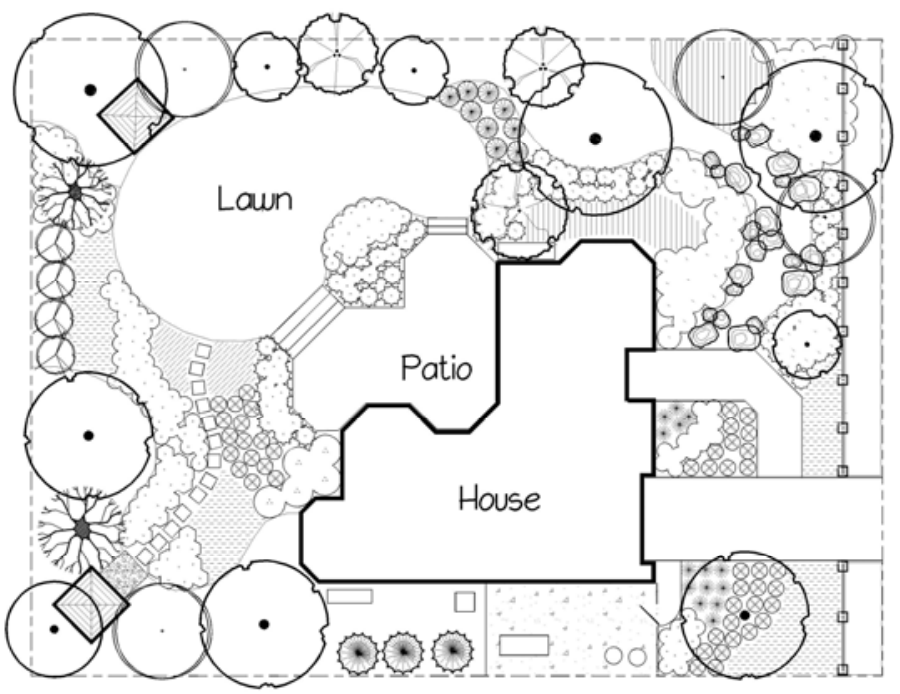

Figure 5. Naturalistic Form Theme. Credits: Gail Hansen

Form themes are sometimes combined; geometric shapes are used for the hardscape and naturalistic shapes for the plantings. For example, plant bedlines are often curvilinear while the hardscape is square in form.
Style themes are most often related to the architecture and they often simplify the design of a residential yard because materials and form are to some extent pre-determined. Many style themes today are a contemporary version of traditional garden designs. Architecture is usually the primary source of a theme, but they can also represent a time, a culture, a place, or a feeling, such as serenity or calmness. The advantage of using a traditional style theme is the established set of forms and elements have historically worked well together and endured the test of time.

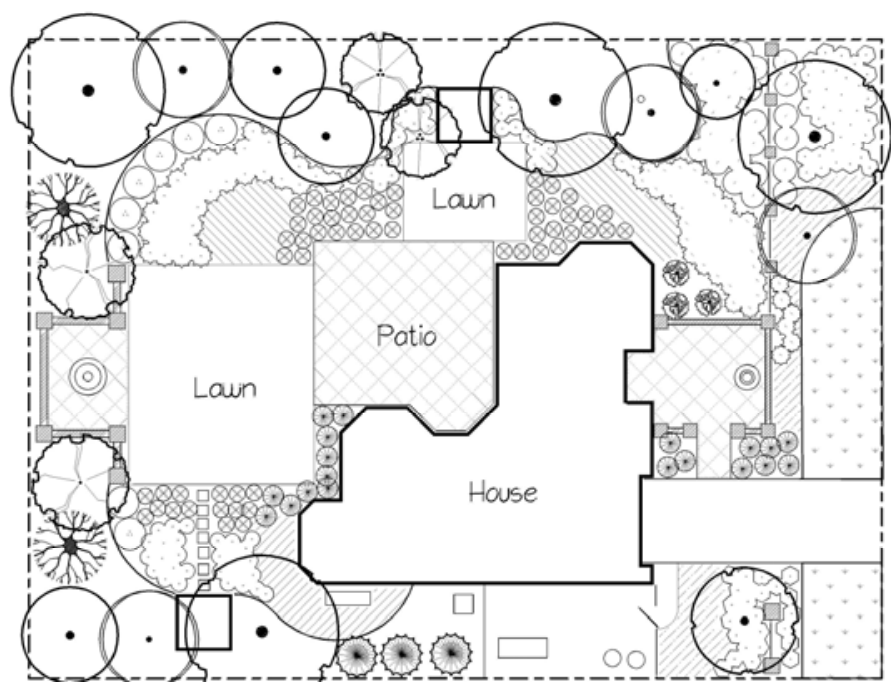

Figure 6. Formal Style Theme. Credits: Gail Hansen

Because architectural styles typically fall into a formal or informal category; the landscape theme tends to be either formal or informal (Figure 6). Formal architectural and garden styles that can be used for inspiration include French, Spanish, Italian, and Middle Eastern. Less formal designs include Oriental, English, and American. Style themes can also apply to the planting plan and include tropical, desert, meadow, woodland, marsh, or coastal plantings. Themes can be as simple as a color mix or plants with a distinct character- such as grasses-used repeatedly in the composition. 


\section{Create and Link Spaces}

The yard is an extension of the home where a variety of activities take place. A yard can generally be divided into three areas: public (the front yard), private (the back yard) and service (typically the side yard). The location of activity areas depends primarily on the type of area, the size of space needed, the type of activity, and the desired proximity to other activities and structures. A few examples of spaces include the front entry area that brings you and your visitors to your home, a cooking/eating/entertaining area (patio or deck), a play area, a dog run, a secret garden/relaxation area, a vegetable garden or hobby area, and a trash/compost/work area.

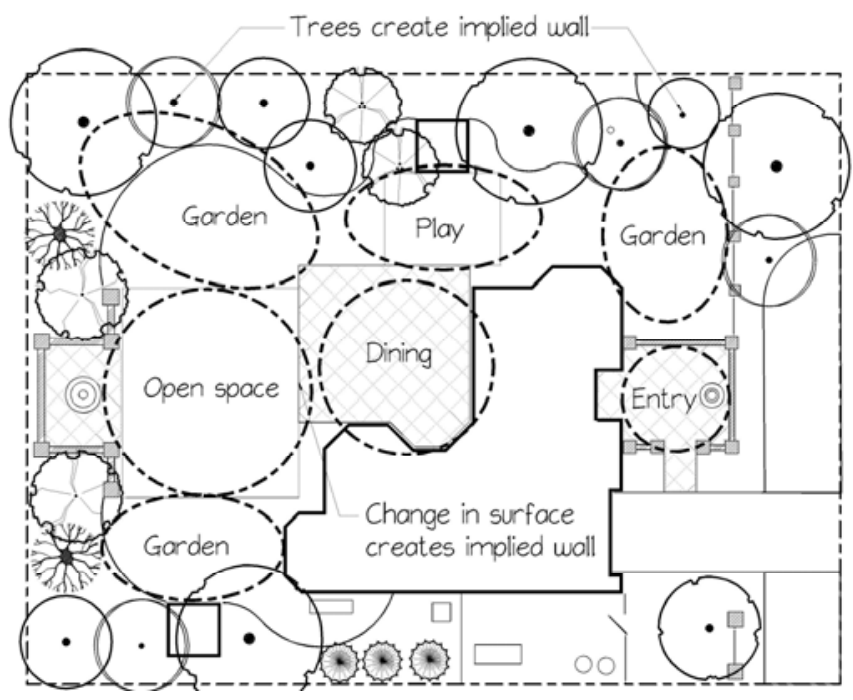

Figure 7. Outdoor Rooms. Credits: Gail Hansen

Perhaps the most important spatial concept for successful garden design is the creation of outdoor rooms in the yard. These spaces are often separated through the use of plant beds, sod areas, trees, planters, garden walls, arbors, level changes, and paved surfaces. (Figure 7) The features are used to enclose or define the spaces and give them a room-like feel. For psychological comfort creating spaces that are human scale is important because most people prefer to be in places that feel protected and sheltering, rather than open and exposed. The outside wall of the house often serves as the first wall or starting point of an outdoor room.
Incompatible uses should be separated, and related activities, such as cooking and dining, should be together to make the yard more efficient and enjoyable. When using hardscape to create spaces use construction material similar to that used in the house for continuity from the house into the garden.

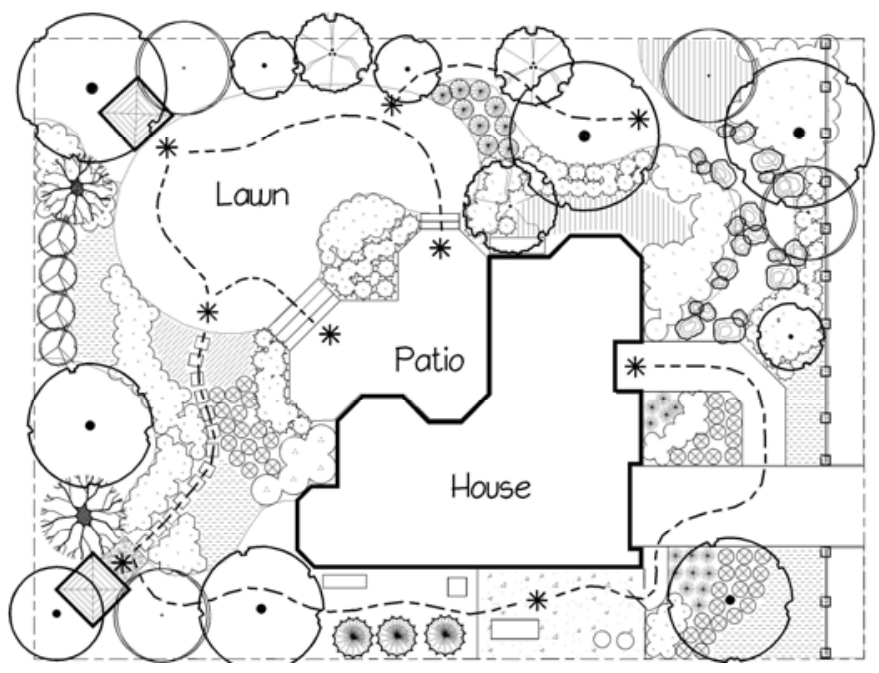

Figure 8. Linked Spaces. Credits: Gail Hansen

Pedestrian circulation in the landscape should move people through the yard and provide organizational structure. Outdoor rooms are typically linked by pathways, steps, and walkways or openings with gates or arbors that encourage exploration and use of the entire yard (Figure 8). These spaces can also be linked by visual features such as a creek bed (wet or dry), that meanders through or beside several spaces, or a garden wall that begins at a patio, moves along a turf area and ends along a planted area. Using similar hardscape features and repeating plants pulls the eye around the garden. Important points along the way can be emphasized with plantings or features that draw attention and encourage movement in a particular direction. Moving along the path takes a person from one area to the next and allows the user to have a variety of experiences. In an informal garden the curves and bends of the path should partially conceal what lies ahead. This provides a sense of mystery that promotes exploration and discovery of the landscape. 


\section{Consider the Function of Plants}

From a design perspective, plant materials have three major functions in the landscape: aesthetic, structural, and utilitarian.

Aesthetically plants create a visually pleasant environment and structurally plants organize and define spaces. Plants are utilitarian because they can transform the environment for the comfort of the user by modifying light, temperature and humidity. Plants can also be used to control noise and odor, and provide food for homeowners and wildlife.

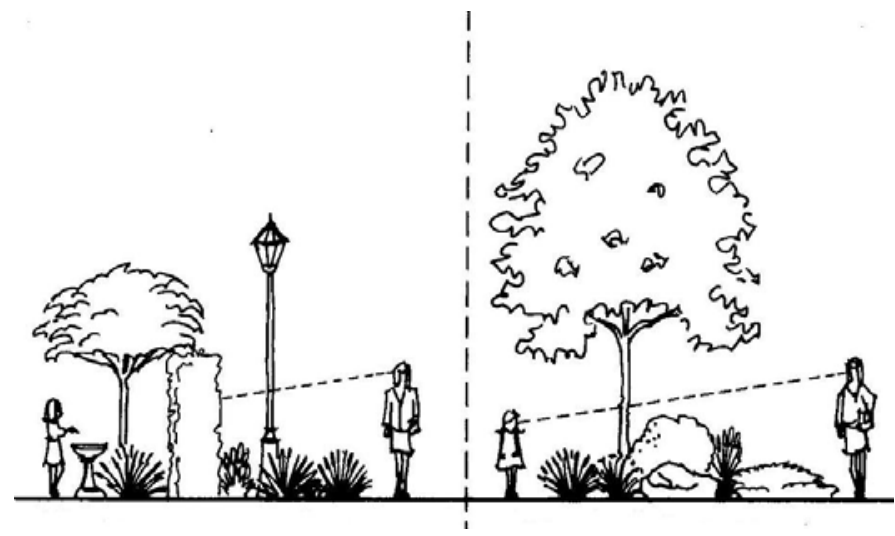

Figure 9. Physical and implied barriers. Credits: Gail Hansen

For psychological comfort plants are used as physical or implied barriers for privacy and safety. Physical barriers block both the view and access to a space and include fences, walls and plant hedges. Implied barriers, typically low growing plants, block access but not the view (Figure 9). Other functions of plants include cleaning the air, preventing erosion and soil loss, retaining moisture in the soil, and returning organic matter to the soil.

For these reasons the type of plants to be used (such as tree, shrub or groundcover) should be chosen in the early stages of planning. Plant types are chosen for their functional capabilities so that their future purpose and required space can be considered at the same time.
For structure and utility trees and large-tomedium shrubs are the most important plants in the landscape, they contribute the most to microclimates (Figure 10), spatial organization,

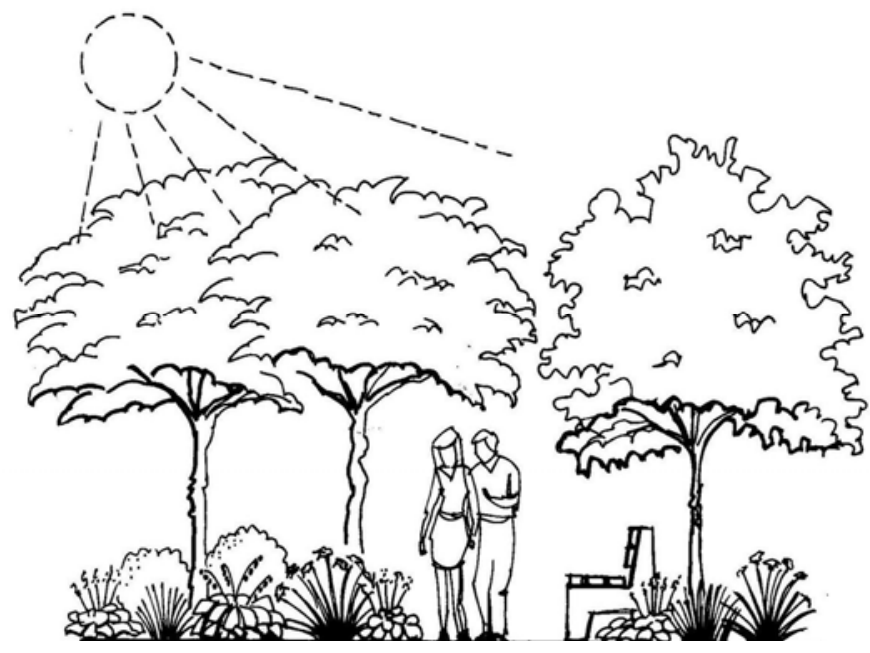

Figure 10. Microclimates. Credit: Gail Hansen

and security concerns. The size, form and texture (density) are the three dominant characteristics that determine how the plant can be used to make more pleasant microclimates and create a more functional, organized, energy efficient yard.

\section{Structure the Plantings}

Plants provide structure in the garden in the same way as the walls of a building. Shrubs can act as walls in a space and the branches of trees form a ceiling canopy overhead. The overhead plane, the vertical plane and the ground plane should all be considered to create enclosure. Openings between plant materials serve as windows and doors. Once the shape of a plant bed has been established, the plants should be massed (grouped) and layered to achieve visual unity and the desired amount of enclosure. The size of a plant mass will depend on the total size of the yard, the size of the individual plants in the mass, and the emphasis or impact desired of the plant material. 
Overlapping the masses of plants so that they are layered, both horizontally (Figure 11) and vertically (Figure 12) links the plants with an interconnecting pattern. Each plant mass is in front of, behind or next to, another mass.

Repeating plants within a mass and repeating masses with similar plants ties the garden together.

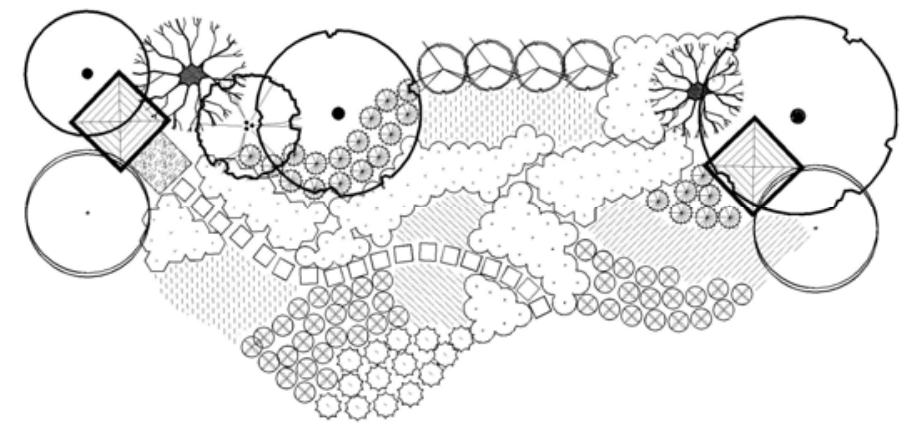

Figure 11. Horizontal plant layers. Credits: Gail Hansen

The individual plant characteristics must be considered to successfully layer and mass plants. Each plant should complement or contrast a

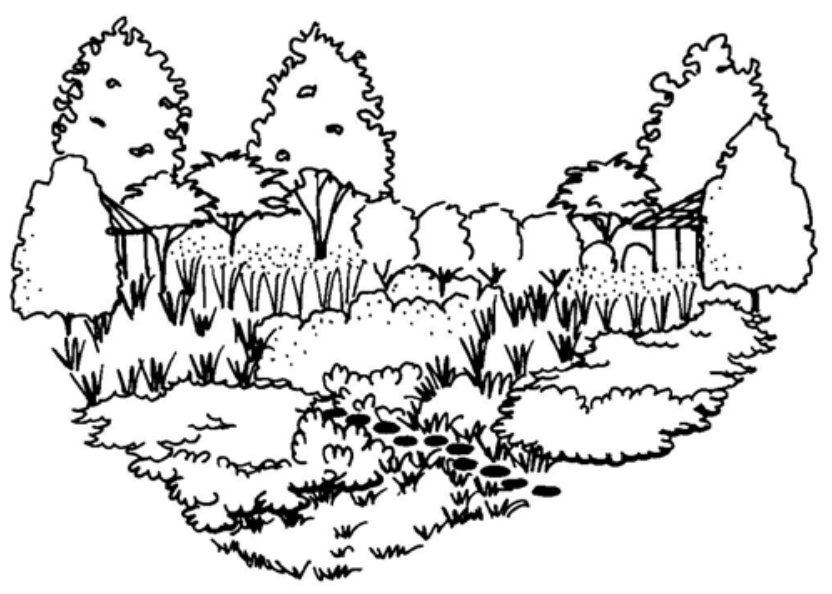

Figure 12. Vertical plant layers. Credits: Gail Hansen

neighboring plant. All plant compositions begin with the main structure plants, the large, mostly evergreen plants- such as trees and large shrubs. These plants separate or enframe spaces, control the size of the space, and provide the starting point for choosing the appropriate characteristics of the second layer, midground plants, for massing and infill. The final layer of plants, the foreground plants, includes the low growing plants that provide emphasis or focal points.

\section{Highlight Important Points}

Important points in the garden should be highlighted by the use of unique plants, distinct structures, or garden ornaments. Marking thresholds or entrances to spaces can be done with gates, arbors, and steps, or through the use of unique and colorful plants. The form and/or style theme of the garden will help determine the important points and how they should be highlighted. Some styles have signature elements, such as a type of statuary or water feature, that are the hallmark of the style. Other important places in the yard are focal points, which is used to visually organize a landscaped area. The type of focal point often depends on the viewing perspective. Different perspectives or viewpoints can reveal different compositions in the landscape that may require a variety of focal points. Contrasting texture, shape, size and color will capture and hold the eye.

\section{Pay Attention to Detail}

Detail in the landscape is derived from the visual quality of plants, hardscape and garden ornaments. Sensual qualities such as scent, sound, and touch also add detail to the landscape. Various combinations of form, color, texture, and size create aesthetic qualities. Plants come in various forms-such as round, columnar, weeping, and spreading, that compliment or contrast with each other depending on how similar or different they are (Figure 13). Form is the most enduring quality of a plant and thus the most important characteristic. After form, texture is the next dominant feature of a plant; coarse, medium and fine textures can be used for contrast and emphasis in the landscape. Form and texture both trump color in the garden for most of the year. However, during certain seasons, color will be the most noticeable characteristic of the garden. To extend color displays throughout the year, use 
plants that have color in numerous plant parts, such as the foliage, bark, and fruit.

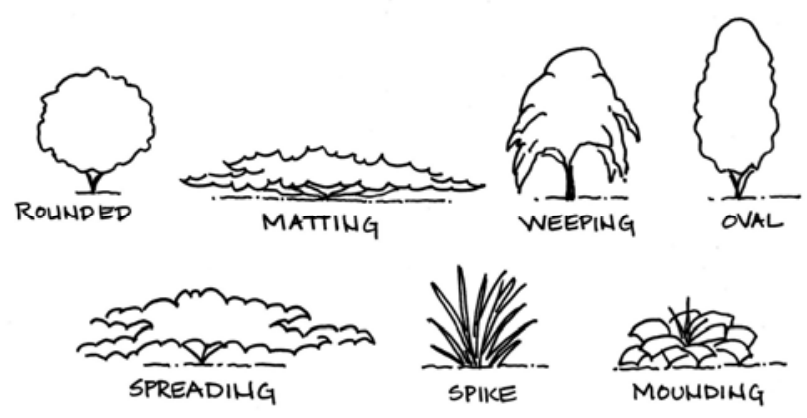

Figure 13. Plant Forms. Credits: Gail Hansen

Various color schemes, based on color theory, are used for contrast or unity in a garden. Typically one color, two contrasting colors, or several analogous (similar) colors are repeated throughout the garden. Both color and texture affect distance and can change the perceived size of a space- warm colors and/or coarse texture make a space feel smaller; cool colors and/or fine texture make a space feel larger. The characteristics of individual plants affect balance, unity and emphasis in the composition. Dark colors and coarse textures make a plant look heavier and larger (more visual weight) and must be balanced with the same or with a larger mass of bright or light colors with fine or medium texture. Massing plants with similar colors and textures provide unity, but contrasting plants should be included to provide emphasis at important points.

To fully appreciate and experience a garden all of the senses must be considered. The pleasant scent of plants, the sound of wind in the trees, the sound and texture of water, and the colors and textures of sculptures, pots and garden furniture all add to the experience of the garden. One detail that is often overlooked is the affect of light on the aesthetics of the plants. Light shining through a plant, such as a feathery grass, can change the appearance of the plant. The entire garden changes in function and appearance over the course of the day, and the course of a year, as the light and temperature changes from morning to night and season to season.

\section{Take Time into Account}

Plant selection must consider a plant's growth rate, its mature size and form, and the maintenance it will require. Fast growing plants may or may not be desirable depending on the size of the site, the design intent, and the expected level of maintenance. It is important to know the eventual mature size of plants so they can be placed in the right location and spaced properly when they are installed. Giving plants room to grow is a challenge because the mature size is typically based on optimal growing conditions and the environmental conditions of a site may cause a plant to grow larger or stay smaller. If plants are spaced too closely (to simulate a fully grown landscape) the overcrowded plants will present a maintenance issue.

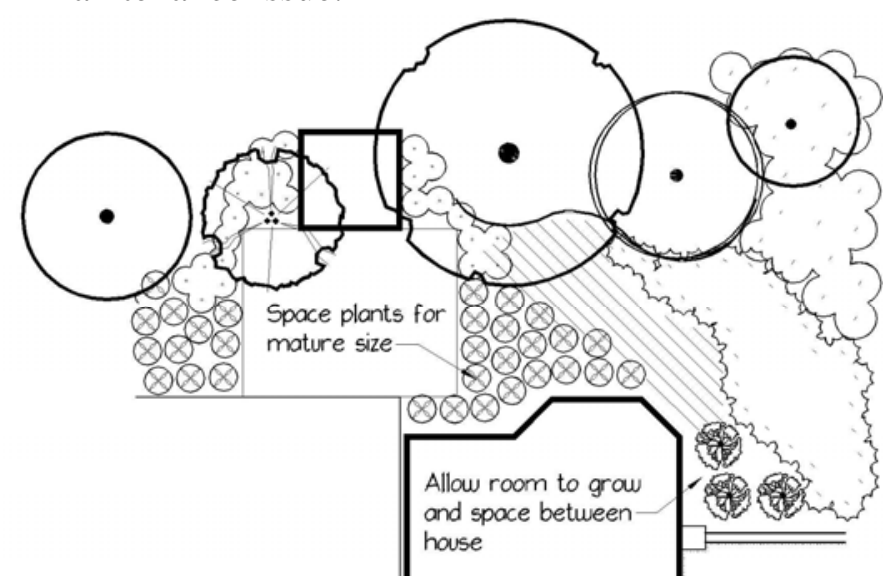

Figure 14. Plant Spacing. Credits: Gail Hansen

Provide enough space so that when fully mature, the plants are barely touching their neighbors and a somewhat solid growing mass is formed (Figure 14). It is important, however, to leave space so the plant does not touch the side of the house- this space allows for air circulation and home maintenance. Another common mistake is to plant too close to utilities such as AC units and meters. 


\section{Protect Your Resources}

Be a good steward of the land through the wise use of water, plants, and building materials. The three areas of greatest impact are the use of resource-efficient plants, the management of water, and the use of environmentally sound hardscape materials. Before you remove any plant material consider how you might be able to enrich your existing ecological capital. That is, look at what you have and decide if it is worth saving. Some plant material may simply be in the wrong place and can be transplanted for more favorable conditions. Look for resource-efficient plants when choosing new plants. Florida-friendly plants are long lived, insect and disease resistant, and don't demand frequent trimming, fertilizing, and watering.

The design phase is the time to consider incorporating the development of a rainwater catchment system. The availability of water will no doubt be limited in the future so you must decide the role of water in your design: where will you get irrigation water, how you will manage it, and how will you use it? The most obvious source will be rainwater and the use of a harvesting system to capture, move, and store it. The system can be an aesthetic design element if planned for early in the process. It is typically the first element to be installed because it sometimes requires earthmoving and underground piping.

Protecting resources also includes the use of environmentally friendly hardscape materials and non-toxic preservatives, stains, paints, and cleaners. Reusing construction materials will reduce the environmental impact of using new materials and keep old materials out of the waste stream. Before any demolition starts on your old patio, pool deck, arbors, or structures, consider how you might reuse the material in your new design. In addition to helping the environment it will also help your budget. If you will not be doing any demolition work in your yard, look for other sources of used material in your community.

\section{Summary}

Your yard is a very important natural resource that adds value to your home and enjoyment to your life. It contributes to a desirable, healthy community. Several important concepts will help you create an aesthetically pleasing, functional, and sustainable design. First remember to put your ideas on paper: create a design plan. Use the design process to identify and understand your site and your needs. Use a theme to guide your decisions; professional designers always look to the existing conditions for inspiration and material and plant choices. Create spaces that serve as outdoor rooms- this is an essential concept of design- both for functionality and psychological comfort human scaled spaces are the most desirable. Use plant material to create the walls, ceilings and floors of the rooms for a physically comfortable microclimate. Remember that plants change over time, plan for this and you will find that observing the changes is one of the most rewarding aspects of your garden. In many of our urban areas private yards represent the last remnants of green space, think about how your yard fits into the big picture and what you can do to have a positive impact on the environment in your neighborhood.

\section{Sources:}

Austin, R. (2002). Elements of Planting Design. John Wiley \& Sons, Inc. New York, NY

Bertauski, T. (2005). Designing the Landscape: An Introductory Guide for the Landscape Designer. Pearson Education, Inc. Upper Saddle River, New Jersey

Booth, N. (1983). Basic Elements of Landscape Architectural Design. Waveland Press, Inc. Prospect Heights, Illinois 
Chaplin, L.T., \& Slack, W. (1998).

Landscaping, Southern Living Garden Guide.

Oxmoor House Inc. Birmingham, Alabama

Erler, C. T. (2007). Creative Homeowner:

Design Ideas for Home Landscaping. Upper

Saddle River, NJ

Holmes, R., \& Buchanan, R. (2006). Creative

Homeowner: Southeast Home Landscaping.

Upper Saddle River, NJ

Levy, C. (2001). Landscaping Your Home, Fine Gardening Design Guides. The Taunton Press, Inc. Newtown, CT

Scarfone, S. (2007). Professional Planting Design: An Architectural and Horticultural Approach for Creating Mixed Bed Plantings. John Wiley \& Sons, Inc., Hoboken, New Jersey

Smith, C., Clayden, A., Dunnett, N. (2008). Residential Landscape Sustainability, A Checklist Tool. Blackwell Publishing, Oxford, UK Etc. 